\title{
Estratégias gastronômicas para melhorar a aceitabilidade de dietas hospitalares:
}

\section{Uma breve revisão}

\author{
Gastronomic strategies to improve acceptability of hospital diets: A brief review \\ Estrategias gastronómicas para mejorar la aceptabilidad de las dietas hospitalarias: Una breve \\ revisión
}

Recebido: 16/04/2021 | Revisado: 23/04/2021 | Aceito: 27/04/2021 | Publicado: 12/05/2021

Crislaine da Costa Fischer

ORCID: https://orcid.org/0000-0003-2023-6881 Universidade do Estado do Rio de Janeiro, Brasil E-mail:crislaine.fischer7@gmail.com

Karen de Oliveira Flor

ORCID: https://orcid.org/0000-0002-9521-4129 Universidade do Estado do Rio de Janeiro, Brasil E-mail:karenflorr96@gmail.com

Lilia Zago

ORCID: https://orcid.org/0000-0002-6821-4991 Universidade do Estado do Rio de Janeiro, Brasil

E-mail: lilia.zago@gmail.com

Roberta Fontanive Miyahira

ORCID: https://orcid.org/0000-0002-4202-2756 Universidade do Estado do Rio de Janeiro, Brasil E-mail: robertamiyahira@gmail.com

\begin{abstract}
Resumo
O agravamento do estado nutricional durante a internação hospitalar, afeta negativamente o estado clínico do paciente, pois, podem ocorrer alterações fisiológicas oriundas de um processo adaptativo do organismo à escassez de nutrientes. Nesse sentido, o presente artigo teve por objetivo discutir estratégias de gastronomia hospitalar voltadas para a aceitação de dietas, com o intuito de auxiliar na prevenção da desnutrição hospitalar. Trata-se de uma revisão integrativa da literatura. Foram realizadas buscas nas bases de dados National Center for Brotecnologia for Information (PUBMED), Scientific Electronic Library Online (SciELO), e em livros da temática, entre o recorte temporal de 1997 e 2021. Os estudos mostraram que a desnutrição hospitalar é uma condição multifatorial, com prevalência entre $20 \%$ a $50 \%$ dos pacientes internados, em âmbito mundial, que aumenta significativamente a morbidade, mortalidade, duração da estadia hospitalar e taxa de readmissão hospitalar. A redução da ingestão alimentar é uma das causas da desnutrição hospitalar, e pode estar associada a baixa aceitação de dietas. Dessa forma, a aplicação de técnicas de gastronomia hospitalar que possibilitem a melhoria das características sensoriais das preparações são ferramentas essenciais para aumentar a aceitação das preparações oferecidas aos pacientes, favorecendo a manutenção e/ou recuperação do estado nutricional, bem como a redução do desperdício de alimentos. Mais estudos sobre essa temática devem ser realizados para que sejam avaliadas e desenvolvidas novas estratégias voltadas para a gastronomia hospitalar aplicadas à dietoterapia.
\end{abstract}

Palavras-chave: Desnutrição; Dietética; Dietoterapia; Pacientes internados; Satisfação do paciente.

\begin{abstract}
The worsening of the nutritional status during hospitalization negatively affects the patient's clinical status, because physiological changes may occur as a result of an adaptive process of the body to the lack of nutrients. In this sense, the present article aimed to discuss hospital gastronomy strategies aimed at the acceptance of diets, in order to help prevent hospital malnutrition. This is an integrative literature review. Searches were made in the National Center for Information Technology (PUBMED), Scientific Electronic Library Online (SciELO) databases, and in books on the subject, between 1997 and 2021. The studies showed that hospital malnutrition is a multifactorial condition, with a prevalence between $20 \%$ and $50 \%$ of inpatients worldwide, which significantly increases morbidity, mortality, length of hospital stay, and hospital readmission rate. Reduced food intake is one of the causes of hospital malnutrition, and may be associated with low diet acceptance. Thus, the application of hospital gastronomy techniques that enable the improvement of sensory characteristics of the preparations are essential tools to increase the acceptance of the preparations offered to patients, favoring the maintenance and/or recovery of the nutritional status, as well as the reduction of food waste. Further studies on this theme should be conducted to evaluate and develop new strategies for hospital gastronomy applied to diet therapy.
\end{abstract}

Keywords: Malnutrition; Dietetics; Diet therapy; Hospitalized patients; Patient satisfaction. 


\begin{abstract}
Resumen
El empeoramiento del estado nutricional durante la hospitalización afecta negativamente al estado clínico del paciente, ya que pueden producirse cambios fisiológicos como resultado de un proceso de adaptación del organismo a la falta de nutrientes. En este sentido, el presente artículo tiene como objetivo discutir las estrategias gastronómicas hospitalarias dirigidas a la aceptación de las dietas, con el fin de ayudar a prevenir la desnutrición hospitalaria. Se trata de una revisión bibliográfica integradora. Las búsquedas se realizaron en las bases de datos National Center for Biotechnology for Information (PUBMED), Scientific Electronic Library Online (SciELO) y en libros sobre el tema, entre los años 1997 y 2021. Los estudios mostraron que la desnutrición hospitalaria es una condición multifactorial, con una prevalencia de entre el $20 \%$ y el $50 \%$ de los pacientes hospitalizados en todo el mundo, que aumenta significativamente la morbilidad, la mortalidad, la duración de la estancia hospitalaria y la tasa de reingreso en el hospital. La reducción de la ingesta de alimentos es una de las causas de la desnutrición hospitalaria, y puede estar asociada a la baja aceptación de las dietas. Así, la aplicación de técnicas de gastronomía hospitalaria que permitan mejorar las características sensoriales de las preparaciones son herramientas esenciales para aumentar la aceptación de las preparaciones ofrecidas a los pacientes, favoreciendo el mantenimiento y/o la recuperación del estado nutricional, así como la reducción del desperdicio de alimentos. Deberían realizarse más estudios sobre este tema para evaluar y desarrollar nuevas estrategias de gastronomía hospitalaria aplicada a la dietoterapia.
\end{abstract}

Palabras clave: Desnutrición; Dietética; Terapia dietética; Pacientes internos; Satisfacción de los pacientes.

\title{
1. Introdução
}

Estudos recentes apontam que o agravamento do estado nutricional durante a internação hospitalar, afeta negativamente o estado clínico do paciente, uma vez que podem ocorrer alterações fisiológicas oriundas de um processo adaptativo do organismo à escassez de nutrientes (Lew et al., 2017; Lima et al., 2010). Como consequência desse processo, o paciente pode cursar com alterações na resposta imunológica, estando mais suscetível a um quadro infeccioso e inflamatório, visto que a desnutrição e o sistema imunológico possuem um sinergismo que leva a potencialização um do outro, agravando ainda mais o estado clínico e nutricional do paciente (Da Silva \& Tiengo, 2014; McCarthy et al., 2019). Além de alterações imunológicas, a desnutrição hospitalar tem como consequências o surgimento de alterações fisiológicas e bioquímicas, que também podem resultar no agravo do quadro clínico do paciente e do seu tempo de internação (Moriana et al., 2013; Saengnipanthkul et al., 2021; Toledo et al., 2018).

Para manutenção e recuperação do estado nutricional, é necessária uma alimentação balanceada e adequada de acordo com as especificidades de cada indivíduo. É comum associar a alimentação servida no hospital à uma comida pouco palatável, visto que as modificações de consistência e composição de nutrientes, necessárias para atender as demandas individuais, podem comprometer o sabor, aroma, textura e temperatura dos alimentos, os quais influenciam no apetite e grau de satisfação do paciente (Da Silva \& Maurício, 2013; Novinski et al., 2017).

Ao longo do tempo, modificações no âmbito da alimentação hospitalar foram ocorrendo com intuito de valorizar práticas que aproximem os pacientes a uma alimentação com maior relação afetiva, promovendo conforto e qualidade, levando em consideração processos que compreendem a produção e distribuição de preparações que vão compor as dietas hospitalares, bem como a relação com o paciente (Martins \& Baratto, 2018).

Nesse contexto, a aplicação de estratégias de gastronomia visa melhorar a qualidade sensorial das preparações servidas no hospital, ao garantir um padrão estético na forma de apresentação do alimento no prato, o uso de condimentos e especiarias, a combinação de ingredientes, e a manutenção da temperatura adequada do alimento, contribuindo para menor índice de rejeição da dieta (Souza, Gontijo \& Almeida 2017). A melhora das características sensoriais das preparações oferecidas e da aceitação da dieta pelo paciente, por meio de intervenções nas técnicas de preparo, na distribuição e apresentação dos alimentos oferecidos, são essenciais para favorecer a adequação das necessidades nutricionais. (Souza \& Nakasato, 2011).

O uso de estratégias de gastronomia hospitalar visa minimizar os efeitos das restrições dietéticas, na tentativa de prover uma alimentação, além de saudável e nutritiva, atraente do ponto de vista sensorial ao paciente, que já se encontra em um ambiente que costuma ser indesejável. A implementação de técnicas gastronômicas, favorecem a aceitação da dieta 
hospitalar, e consequentemente contribuem para a melhora do estado nutricional do paciente internado (Souza \& Nakasato, 2011).

O objetivo deste estudo foi discutir, através da revisão integrativa de literatura, estratégias de gastronomia hospitalar voltadas para a aceitação de dietas, com o intuito de auxiliar na prevenção da desnutrição hospitalar.

\section{Metodologia}

Trata-se de uma revisão integrativa da literatura, de caráter qualitativo e exploratória, construída a partir de análises de livros, artigos publicados em periódicos e em bases de dados específicas, que abordam a temática e exploram suas evidências. A elaboração do artigo seguiu as seguintes etapas: análise e reflexão da hipótese, objetivos, critérios de inclusão e exclusão para o levantamento bibliográfico, arguição do problema, resultados e discussão e conclusão da temática abordada.

O levantamento bibliográfico, foi elaborado a partir de estudos disponíveis nas bases de dados: National Center for Biotecnologia Information (PUBMED) e Scientific Electronic Library Online (SciELO). De modo complementar às buscas, utilizou-se livros impressos e bases eletrônicas. As buscas foram iniciadas em agosto de 2020 e finalizadas em março de 2021.

Ao longo do levantamento, utilizando-se nos campos de busca de "título", "assunto" e "palavras-chaves, os seguintes descritores para seleção: "Hospital-Acquired Malnutrition", "Nutritional Intervention”, "Diet Acceptance", "Sensory Quality” e "Hospital Gastronomy", cruzando-os pelo uso do operador booleano "AND".

Ao longo da pesquisa inicial, encontrou-se 644 publicações nas bases de dados, sendo 34 trabalhos na base de dados SCIELO, 610 trabalhos no PUBMED, 4 livros e 4 artigos de bases eletrônicas. Para seleção das publicações, foram criados critérios de inclusão dos artigos durante o levantamento, sendo: artigos completos disponíveis nos idiomas inglês, espanhol e português, publicados entre os anos de 1997 e 2021, nas bases de dados citadas, e que abrangessem a temática da desnutrição hospitalar e os impactos das ações de gastronomia hospitalar sobre o estado nutricional de pacientes hospitalizados. E como critério de exclusão dos estudos, utilizou-se artigos nos demais idiomas, publicações realizadas em um recorte temporal abaixo do ano de 1997 e os artigos que fugissem da temática a ser estudada. Assim, os artigos e livros obtidos pela estratégia de busca, foram filtrados primeiramente pela leitura de seus títulos, em seguida o seu idioma, depois a partir da data de publicação e por fim, foi avaliado a adequação e pertinência do tema. Após a triagem e análise dessas publicações, baseado nos critérios de inclusão e exclusão, foram excluídas manualmente aquelas que não eram pertinentes aos critérios. Dessa forma, a amostra final obtida foi, 7 publicações da base de dados SCIELO, 45 publicações do PUBMED, 4 livros e 4 artigos de bases de eletrônicas.

Por fim, todos os artigos e livros selecionados, após aplicação dos critérios, foram lidos em sua totalidade, a fim de estabelecer dados e materiais de análise relevantes para a construção do artigo.

\section{Resultados e Discussão}

\subsection{Definição e etiologia da desnutrição hospitalar}

Segundo a Organização Mundial da Saúde (OMS), a desnutrição é caracterizada por um desequilíbrio celular que ocorre entre a demanda energética e os nutrientes fornecidos para o seu crescimento, manutenção e funções específicas essenciais para o funcionamento do organismo (WHO, 1997). A desnutrição, incluindo a desnutrição em ambientes hospitalares, continua a ser uma questão global (Syuhada et al., 2020). Na literatura, o termo desnutrição hospitalar tende a ser utilizado de forma semelhante ao termo desnutrição hospitalar adquirida (Cheng et al., 2019). A falta de critérios estabelecidos para o diagnóstico de desnutrição acrescenta mais complexidade à abordagem desta questão, uma vez que não é fácil definir com precisão a prevalência da desnutrição entre os pacientes no momento da admissão no hospital (Orlandoni et al., 2018).

Esse fato dificulta distinguir se a causa da desnutrição teve origem no hospital ou se o paciente já se encontrava desnutrido no momento da admissão (Cheng et al., 2019). A desnutrição observada em pacientes hospitalizados é 
frequentemente uma combinação de caquexia (relacionada com doenças) e má nutrição (consumo inadequado de nutrientes) (Barker, Gout \& Crowe, 2011).

A desnutrição hospitalar, é um agravo multifatorial que atinge o estado nutricional e de saúde dos pacientes e que está relacionada a sintomas clínicos adversos (Lew et al., 2017). Portanto, a desnutrição aumenta significativamente a morbilidade, mortalidade, duração da estadia hospitalar e taxa de readmissão hospitalar (Theilla et al., 2015).

Na Figura 1 pode-se observar que a desnutrição hospitalar pode estar associada a diversas causas que resultam na redução do consumo alimentar e do aumento do catabolismo energético, tais como anorexia, baixa aceitação à dieta, alteração no processo de digestão e absorção dos alimentos e nutrientes, estresse metabólico relacionado a doença, capacidade reduzida de deglutição ou ingestão dos alimentos ocasionados por problemas de mastigação, mucosite, sintomas gastrointestinais e também infecções (Beser et al., 2018; Leandro-merhi et al., 2015; Lorenzo et al., 2011; Lovesley et al., 2019; Maeda et al, 2019).

Figura 1 - Principais causas de desnutrição hospitalar.

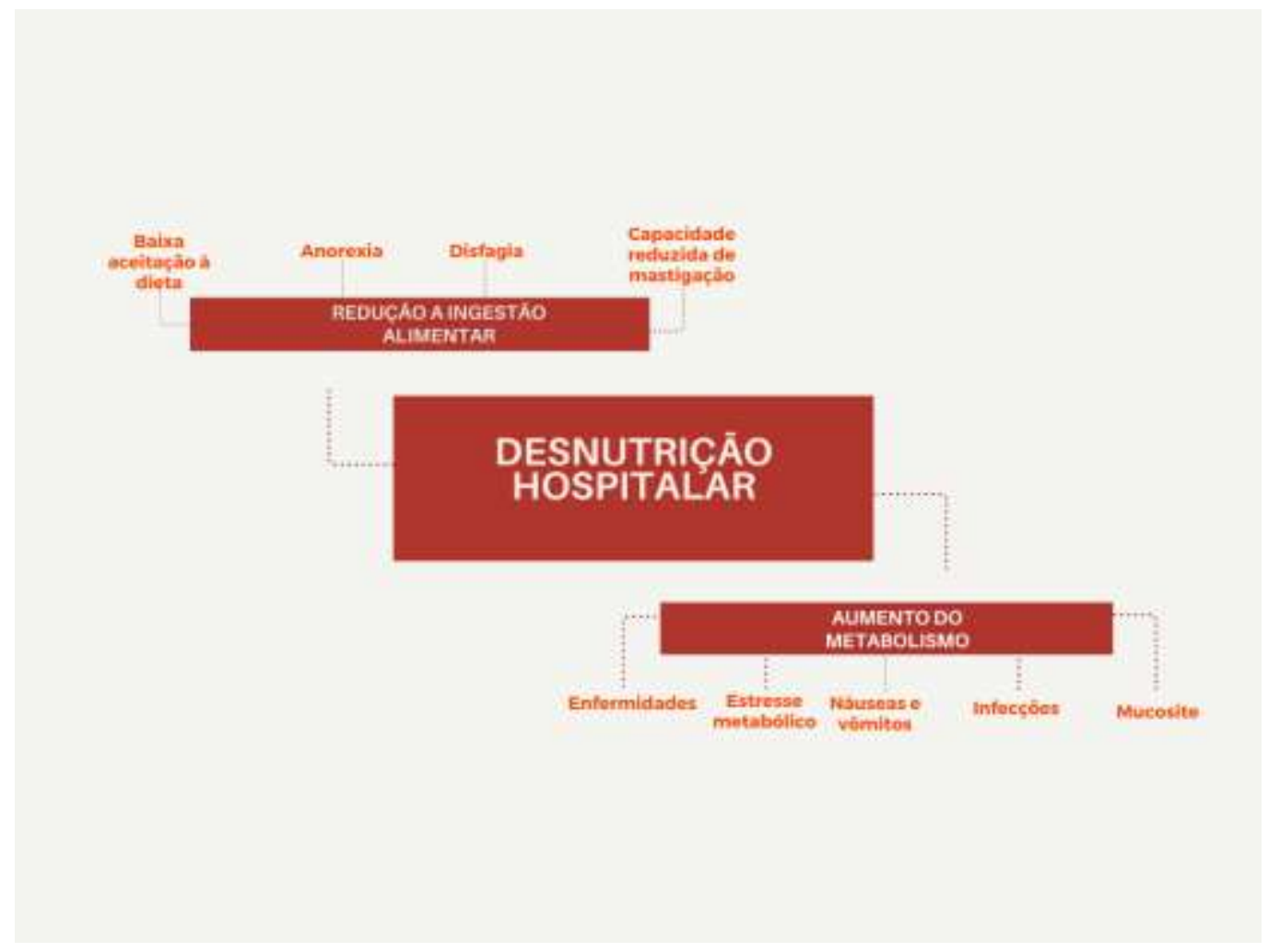

Fonte: Autores.

\subsection{Prevalência de desnutrição hospitalar}

Apesar dos avanços da terapia nutricional na última década, a literatura científica evidenciou que a prevalência de desnutrição hospitalar, em âmbito mundial, é de $20 \%$ a $50 \%$ entre os pacientes, e que esse agravo pode ser caracterizado como um dos fatores responsáveis pelos maiores índices de mortalidade e complicações (Correia et al., 2017; Kang et al., 2018). Esse cenário de prevalência de desnutrição hospitalar, se expressa de modo semelhante entre países desenvolvidos e em desenvolvimento, presentes no território europeu, americano e sul-americano, refletindo então taxas elevadas de desnutrição hospitalar (Barker, Gout. \& Crowe, 2011; Correia et al., 2017).

Em âmbito mundial, um estudo transversal multicêntrico realizado com 99 pacientes de seis hospitais coreanos em 2013, conduzido pelos grupos de pesquisa clínica da Sociedade Coreana de Nutrição Parenteral e Enteral (KSPEN), teve por 
objetivo determinar, por meio dos métodos de Mini Avaliação Nutricional (MNA) e Avaliação Subjetiva Global (ASG), se a prevalência da desnutrição hospitalar da população está associada a desfechos clínicos. O estudo constatou que a taxa de desnutrição hospitalar varia de acordo com o motivo de internação, sendo que essa taxa se apresentou superior (32,5\%) entre pacientes internados para tratamentos médicos, se comparada a pacientes internados para investigação diagnóstica (23,3\%) e cirurgia eletiva $(7,0 \%)$ no país. O estudo também concluiu que entre os pacientes hospitalizados, $81,8 \%$ apresentaram desnutrição moderada, $18,2 \%$ apresentaram desnutrição grave e que a taxa de desnutrição entre pacientes idosos (> 70 anos) foi superior $(38,2 \%)$ do que em pacientes mais jovens (17,2\%) (Lew et al., 2017).

Um estudo australiano em 2009 realizado em um hospital universitário em Melbourne, relatou que a desnutrição foi identificada em $23 \%$ dos 275 pacientes avaliados. Os autores constataram que os pacientes desnutridos apresentaram um período de internação significativamente mais longo em 4,5 dias, em comparação com pacientes eutróficos (Barker, Gout. \& Crowe, 2011). Outro estudo realizado por Konturek et al, (2015) avaliou a prevalência de desnutrição em 815 pacientes internados em um hospital universitário na Alemanha em um período de 12 meses. Os resultados revelaram que a prevalência de desnutrição foi de $53,6 \%$.

Correia et al, (2017) avaliaram estudos de prevalência de desnutrição hospitalar conduzidos desde 2000 em países da América Latina, entre eles Argentina, Brasil, Chile, Costa Rica, Cuba, República Dominicana, Equador, México, Panamá, Paraguai, Peru, Porto Rico, Venezuela e Uruguai. Os resultados demonstraram que cerca de 50\% de todas as pessoas admitidas nos hospitais nesses países apresentaram algum grau de desnutrição.

A Sociedade Europeia de Nutrição Clínica e Metabolismo (ESPEN), em parceria com a Sociedade de Nutrição Clínica da Áustria (AKE) e a Universidade de Viena, criou em 2006, o nutritionDay (nD), que é uma iniciativa para identificar e combater a desnutrição hospitalar, caracterizada por uma ação anual, realizada em um único dia, que avalia o estado nutricional, incluindo ingestão alimentar, de pacientes hospitalizados em países de quase todos os continentes (Theilla et al., 2015). O nD é uma ferramenta reconhecida e valorizada capaz de fornecer informações relacionadas a nutrição de forma instantânea, e os resultados vem contribuindo ao longo dos anos para uma melhor compreensão do impacto da ingestão alimentar abaixo do ideal nos desfechos clínicos de pacientes hospitalizados após 30 (enfermaria) e 60 (UTI) dias da coleta dos dados (Cardenas et al., 2021).

Em nível nacional, um estudo pioneiro chamado Inquérito Brasileiro de Avaliação Nutricional Hospitalar (IBRANUTRI), realizado em 1998 pela Sociedade Brasileira de Nutrição Parenteral e Enteral (SBNPE), evidenciou alta prevalência de desnutrição hospitalar em pacientes no Brasil. Dentre os 4000 pacientes internados avaliados no estudo, observou-se que 48,1\% dos pacientes hospitalizados apresentavam desnutrição hospitalar. Além disso, esse estudo constatou, que os pacientes hospitalizados com desnutrição apresentaram um período de internação e recuperação maior, quando comparados com pacientes sem desnutrição (SBNPE, 2011; Waitzberg, Caiaffa \& Correia, 2001).

O Brasil participa do NutritionDay desde 2009, sendo as ações anuais coordenadas pela Sociedade Brasileira de Nutrição Parenteral e Enteral, que objetivam determinar a prevalência de sintomas, promover o conhecimento e a conscientização sobre a desnutrição hospitalar (Schindler et al., 2014). Os estudos que realizaram o desdobramento dos resultados do NutritionDay no Brasil, em 2015 no Rio Grande do Sul (Pelotas-RS) e em 2016 na Bahia (Salvador-BA), relatam que a maioria dos pacientes adultos e idosos, em 2015 apresentavam eutrofia. Já em 2016, 46,2\% dos pacientes adultos apresentaram sobrepeso, e 50\% dos idosos participantes apresentaram eutrofia (Theilla et al., 2015; Zheng et al., 2016). Os estudos ressaltam que a taxa de desnutrição foi baixa, se comparada ao estudo semelhante realizado na China, onde $29,3 \%$ da população estudada apresentava desnutrição (Theilla et al., 2015). É importante ressaltar que há escassez de estudos que abordem os resultados obtidos pelas ações no NutritionDay no Brasil. 


\subsection{Aceitação de dietas hospitalares}

A nutrição exerce função relevante para o restabelecimento da saúde de pacientes hospitalizados, sendo então necessário a adequação dos diferentes tipos de dieta às especificidades de cada paciente (Rattray, Desbrow, \& Roberts, 2017, Novinski et al., 2017). Para que o planejamento dietético seja feito de forma correta deve-se avaliar a via de administração mais adequada ao paciente. Os alimentos podem ser ofertados pela via oral, quando os pacientes recebem a alimentação pela boca de forma voluntária e não possuem nenhuma contra indicação para o uso do trato gastrointestinal (TGI). A via de administração enteral por tubos, sondas ou ostomias localizadas em alguma porção do tubo digestivo é indicada quando o paciente mantém a ingestão oral insuficiente ou apresente algum distúrbio que torne o TGI parcialmente funcionante. Para indivíduos que apresentam restrição da utilização das vias oral e enteral é indicado o uso da nutrição parenteral, que visa fornecer nutrientes através de acessos venosos, podendo ser acesso venoso central ou periférico (Ross et al., 2016).

Conjuntamente a avaliação sobre a via de administração, verifica-se também qual a melhor dieta a ser oferecida ao paciente, a depender de seu estado de saúde, demanda energética e capacidade de ingestão. O planejamento dietético pode ser elaborado de diversas formas, sendo que a dieta geral é comumente utilizada para pacientes que não necessitam de nenhuma restrição ou limitação. As dietas classificadas como terapêuticas, partem das modificações propostas a dieta geral, e podem apresentar alterações na consistência (dieta líquida, semilíquida, pastosa, branda), adição ou diminuição de algum nutriente (dieta hipossódica, dieta rica em fibras, dieta rica em potássio), restrição de nutriente específico (dieta sem glúten, dieta sem lactose, dieta para alergia), adequação no balanço de macronutrientes (dieta para diabetes, dieta cetogênica, dieta para doente renal, dieta hipolipídica, dieta hiperproteica) (Caruso, Simony \& Silva, 2005; Marliere et al., 2016, Mahan \& Raymond, 2018).

Como mencionado anteriormente, a redução da ingestão alimentar é uma das causas da desnutrição hospitalar, e pode estar associada a diversos fatores, como por exemplo a baixa aceitação de dietas. Apesar da preocupação sobre o estado nutricional de pacientes internados, pouca atenção ainda é dada para a aceitação de dieta hospitalar (Ferreira, Guimarães \& Marcadenti, 2013).

Diferentes métodos podem ser utilizados para avaliar a aceitação das refeições pelos pacientes internados, sendo alguns deles: método de imagem digital - aquisição de imagem, análise através de questionário específico de satisfação, análise do percentual ingerido, através do índice de resto ingestão ou aplicação de questionário de escala hedônica facial, misto ou verbal (Navarro et al., 2016; Coloço, Holanda \& Portero-McLellan, 2009; Fernández-Martínez et al., 2013; Toledo et al.,2018, Augustini et al., 2008).

Indicadores como aparência, sabor, textura, aroma, variedade e temperatura são essenciais para avaliar a aceitação da dieta pelos pacientes. Um estudo realizado por Ferreira et al., (2013) com 100 pacientes internados em uma unidade oncológica de um hospital, teve por objetivo avaliar a aceitação da dieta pelos pacientes. O estudo revelou que as principais queixas relacionadas as dietas foram a falta de sabor $(40 \%)$, a monotonia das preparações (33\%), a quantidade exagerada (29\%) e a temperatura inadequada da refeição (24\%).

Rolim et al., (2011) avaliaram a influência da forma de apresentação das refeições (em bandeja ou em prato) sobre o Índice de resto-ingestão de pacientes oncológicos hospitalizados. As refeições servidas em bandejas tiveram uma média de índice resto-ingestão de $27 \%$ e as refeições servidas em pratos tiveram a média de índice resto-ingestão de 19\%. Os alimentos servidos na bandeja ficavam dispostos separadamente, cada preparação ficava isolada num determinado compartimento da bandeja, enquanto os alimentos dispostos no prato contribuíram para melhor aparência da refeição, permitindo maior harmonia entre os ingredientes utilizados para compor a preparação. Para os autores, a aparência da refeição no prato contribui para o menor índice de resto-ingestão quando comparada a refeição servida na bandeja.

Al-Torky et al. (2016) realizaram um estudo com o objetivo de determinar a satisfação dos pacientes internados com o serviço de alimentação do Hospital Universitário em Sohag, Egito. Foram entrevistados 1015 pacientes, dos quais 35,8\% dos 
pacientes avaliaram a dieta hospitalar como insatisfatória. Do total de pacientes, 26,1\% relataram ter ingerido toda a quantidade servida, 34,7\% consumiram somente parte da quantidade servida e 39,2\% relataram não ter ingerido nada da comida hospitalar. Os motivos para a insatisfação com as preparações relatados foram: os pacientes relataram que sentiram fome entre as refeições e pediam alimentos externos ao hospital (24,6\%), quantidade inadequada (18,7\%), a forma de cocção não era agradável (16,3\%), não gostavam da comida do hospital (14,2\%), a comida hospitalar não era adequada para sua condição clínica $(8,8 \%)$, tinham interesse em outros tipos de alimentos $(6,6 \%)$, outras razões $(10,8 \%)$. Para os autores, esses dados expressam a necessidade de implementar melhorias no serviço de alimentação para aumentar o grau de satisfação dos pacientes internados.

A restrição do sódio é uma alteração que influencia significativamente na aceitação da refeição. As preparações hipossódicas, comumente utilizadas em pacientes com hipertensão, cardiopatias e nefropatias, podem favorecer o menor grau de satisfação quando comparadas à dieta geral (Sousa, Alencar \& Trindade, 2014). Um estudo realizado em um hospital público em Goiânia, com pacientes adultos e idosos com prescrição de dieta hipossódica, verificou por meio da técnica de observação direta que mais da metade dos pacientes não ingeriam todos os alimentos oferecido, no almoço (51,5\%) e no jantar $(60,6 \%)$. Além disso, foi aplicado um questionário para a avaliação da dieta hospitalar, que identificou que 18,2\% dos pacientes consideraram que a dieta hipossódica não apresentava sabor agradável (Casado \& Barbosa, 2015).

Outro estudo realizado em hospitais públicos em Goiânia por Mendes et al., (2019) teve como objetivo avaliar a satisfação dos pacientes com relação às dietas hospitalares oferecidas, identificando quais causam maior insatisfação e quais são satisfatórias. A partir da realização da análise sensorial das dietas livre, branda, pastosa e líquida, com sal e sem sal, foram atribuídas médias para identificar a aceitabilidade. As preparações isentas de sódio obtiveram média insatisfatória quando comparadas às dietas com sal no quesito "sabor/tempero". A menor média corresponde aos atributos sensoriais "aparência" e "odor/cheiro" encontrados na dieta líquida; e a maior média corresponde ao item "quantidade oferecida", encontrado na dieta livre. Seguido da dieta pastosa que apresentou menor média nos quesitos "sabor/tempero", "odor/ cheiro" e "temperatura". Conclui-se a necessidade de adotar medidas para aumentar o consumo das dietas, a implantação de técnicas gastronômicas como uso de ervas aromáticas e condimentos seria uma alternativa para tornar as preparações hipossódicas mais atrativas.

Rigo et al., (2020) realizaram um estudo com homens e mulheres internados em duas unidades hospitalares, com objetivo de avaliar a aceitabilidade das refeições do almoço de pacientes que receberam a dietética para Diabetes Mellitus (DM) e verificar aspectos que podem interferir nesse consumo, como as estações do ano. A pesquisa foi realizada em duas etapas, a primeira etapa foi no verão e a segunda etapa no inverno. Com relação às refeições, observou-se que na primeira etapa, os alimentos menos consumidos foram arroz branco, brócolis cozido, feijão preto e carne bovina; e na segunda etapa, os menos consumidos foram carne bovina, frango, feijão preto e arroz branco. As preparações com maior índice de rejeição foram feijão preto, brócolis cozido, carne bovina, arroz branco, berinjela, cenoura e chuchu cozidos. O desperdício nas refeições do almoço apresentou média per capita de $64,15 \mathrm{~g}$ no verão e de $135,99 \mathrm{~g}$ no inverno. A aceitação de frutas e sucos foi maior no período do verão (82\%) quando comparado ao período do inverno (63\%). Os autores identificaram que esses foram os alimentos que mais se repetiam no cardápio, sugerindo que a falta de variedade das preparações pode causar desinteresse pela refeição.

Fernandes \& Spinelli (2020) avaliaram a percepção de pais e responsáveis por crianças diagnosticadas com câncer quanto à alimentação oferecida no ambiente hospitalar no que se refere à gastronomia hospitalar. Para 36,9\% das crianças, o ambiente hospitalar trouxe a inclusão de alimentos não habituais. Cerca de $88 \%$ dos entrevistados consideraram que a temperatura adequada é um fator importante para aceitação da dieta, outros quesitos apontados foram adequação da quantidade servida, refeições adaptadas às preferências dos pacientes, refeições adaptadas aos sintomas, aparência do prato, e o prato em que é servida a refeição. 
Silva et al. (2021) avaliaram o índice resto-ingestão de 50 pacientes internados em um hospital no Rio de Janeiro. Foi observado que o jantar foi a refeição com maior índice de rejeição (21,5\%), seguido do café da manhã (20,4\%), lanche (14,7\%) e a colação que teve o menor índice de rejeição $(10,6 \%)$. Obtendo um índice resto-ingestão médio de $22 \%$. A falta de padronização no porcionamento do almoço e do jantar, pode ter favorecido o oferecimento de uma quantidade de alimentos maior do que a planejada, o que deve ter contribuído para o índice de rejeição elevado. Em relação aos atributos sensoriais foram avaliados textura, aparência, sabor e odor do almoço e jantar de 12 cardápios. Quanto à textura, o prato proteico foi inadequado para $16,7 \%$ dos entrevistados no almoço e para $25 \%$ no jantar, sendo classificada como uma preparação "dura" dificultando a mastigação. No que diz respeito a aparência, 20,8\% dos entrevistados consideraram que a sopa semilíquida e o feijão não atendiam às características da preparação, pois apresentavam uma coloração amarela e um sabor acentuado de alho, respectivamente. No atributo sabor, houve inadequação para feijão, arroz, prato proteico, guarnição e para as sopas, sendo relacionadas a baixa quantidade de sal e temperos. Todas as preparações foram consideradas adequadas no atributo odor.

\subsection{Implementação de estratégias de gastronomia hospitalar}

A dietoterapia quando aliada ao fornecimento de refeições que geram sensação de prazer ao se alimentar, contribui para que o paciente se sinta acolhido, tendo assim maior interesse pela alimentação, favorecendo a aceitação da dieta (Taldivo \& Santos, 2016). A implementação de gastronomia no ambiente hospitalar, é uma forma de promover o atendimento humanizado, onde o paciente pode ter suas preferências alimentares consideradas, sempre que possível (Pinto \& Alves, 2020), aproximando o paciente do serviço de alimentação e permitindo a ampliação do cuidado nutricional (Oliveira, Oliveira \& Santos, 2020), tornando a refeição assim mais atrativa aos olhos, olfato e paladar (García \& La Hera, 2018).

Como já mencionado, as características sensoriais das preparações são essenciais para avaliar a aceitação da dieta pelos pacientes, e a partir dos resultados pode-se planejar e aplicar as estratégias gastronômicas cabíveis a cada tipo de preparação (Nascimento et al., 2017).

A utilização de técnicas culinárias, mesclando diferentes tipos de cortes (ex.: o fracionamento dos vegetais em formatos decorativos, o corte da carne), variando o método de cocção (diferentes combinações de texturas), combinando as cores dos alimentos no prato (maior variedade de alimentos), e com acréscimo de especiarias (como salsinha desidratada, manjericão, semente de gergelim, cebola em flocos, mostarda em grão), proporcionam uma refeição mais atrativa, contribuem para uma refeição mais saborosa, aumentando a satisfação dos pacientes e a adesão a dieta (Da Silva \& Maurício, 2013). Na Figura 2 observa-se as principais estratégias de gastronomia que podem ser utilizadas para melhorar a aceitação de dietas hospitalares. 
Figura 2. Principais estratégias de gastronomia utilizadas para melhorar a aceitação das dietas hospitalares.

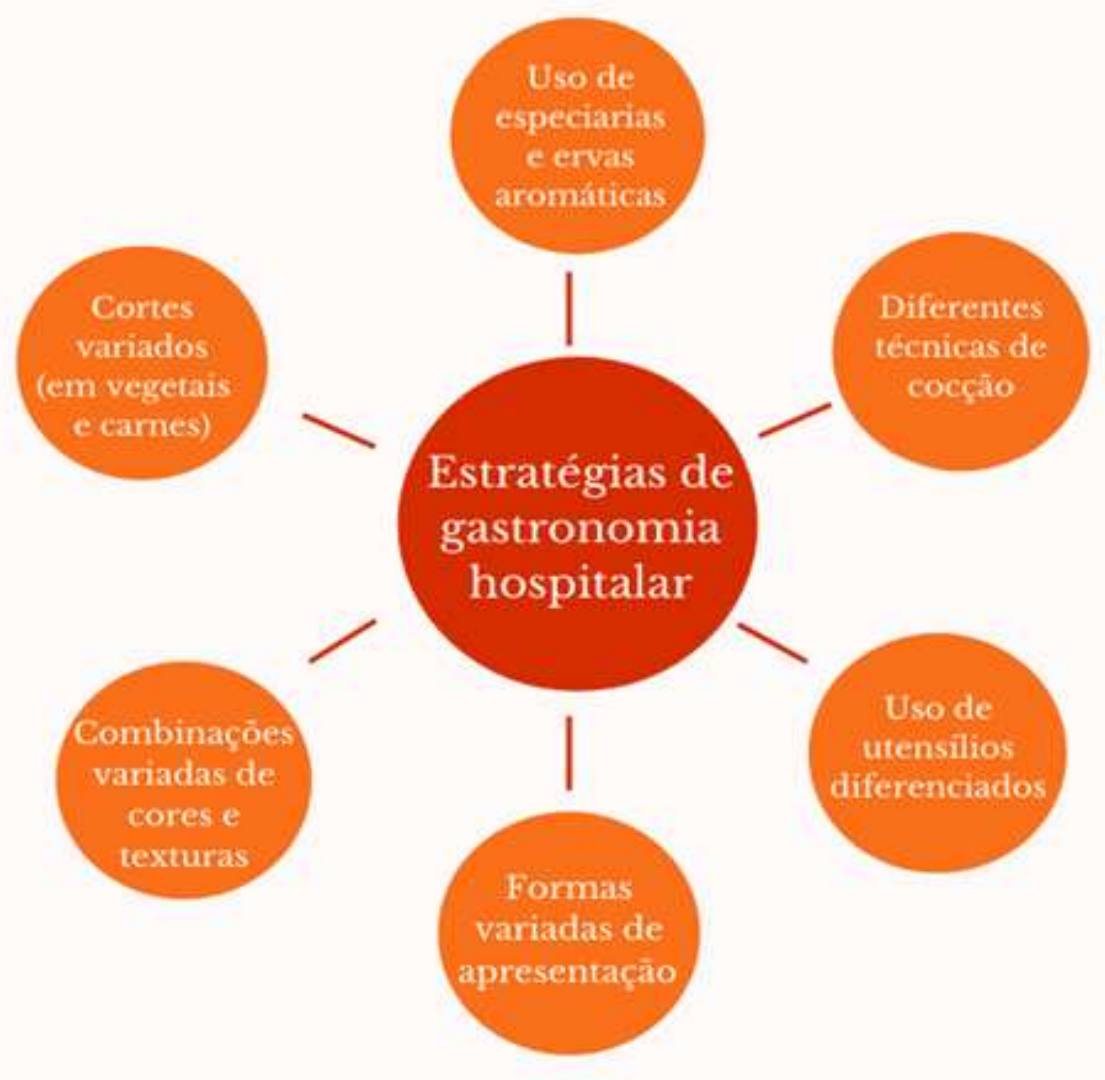

Fonte: Autores.

Navarro et al., (2016) concluíram que a aplicação de técnicas gastronômicas que modifiquem a forma de apresentação dos alimentos dispostos no prato, tornando-o mais atrativo, contribui significativamente para a ingestão de alimentos e para o aumento do desejo de alimentar-se em pacientes internados. Os autores relataram que por meio de aplicações simples de técnica dietética, mantendo os ingredientes comumente utilizados, já é possível melhorar a ingestão alimentar, sem realizar investimentos extras e elevação dos custos.

Um estudo realizado com pacientes oncológicos com prescrição de dieta pastosa internados em um hospital filantrópico localizado em Minas Gerais, teve como objetivo aplicar técnicas gastronômicas para aumentar a satisfação dos pacientes hospitalizados. Foi observado um aumento na aceitação de purês após a implementação de técnicas baseada na elaboração de decorações comestíveis. As intervenções aplicadas foram capazes de melhorar a aparência e textura das preparações. Além disso, a combinação de cores no prato melhorou o aspecto da refeição e aumentou a aceitação do paciente. Anteriormente à aplicação de estratégias de gastronomia, apenas $43 \%$ dos pacientes consideravam as preparações boas. Após a intervenção, $57,1 \%$ dos pacientes passaram a considerar as refeições como boas. Por fim, esse estudo ressaltou a importância da padronização das preparações dietéticas através da elaboração das fichas técnicas de preparação, evitando assim diferenças na refeição quando preparadas por profissionais diferentes (Lages, Soares \& Ribeiro, 2013).

Um estudo realizado com 97 pacientes com idade entre 18 e 59 anos, internados em um hospital filantrópico brasileiro, avaliou a aceitabilidade da dieta servida e de preparações após aplicação de técnicas gastronômicas através do índice resto-ingestão e da aplicação de questionários de escala hedônica. As seguintes técnicas de gastronomia foram aplicadas nas preparações: variação nos tipos de corte (exemplo: jardineira, macedoine, camponesa, mirepoix), utilização de diferentes tipos de cocção (calor úmido, calor, seco e calor misto), utilização de ervas aromáticas, apresentação e combinação de cores. Antes 
da aplicação das técnicas o percentual de pacientes com índice resto-ingestão considerado adequado era de 17,53\% e após o emprego das técnicas de gastronomia esse percentual de adequação subiu para 88,66\%. Com relação aos atributos sensoriais, as variáveis cor, sabor, aroma, textura e temperatura tiveram as notas atribuídas aumentadas após o emprego das técnicas citadas (Souza, Gontijo \& Almeida 2017).

Da Silva e Maurício (2013) realizaram um estudo com objetivo de aplicar técnicas da gastronomia na dieta normal de uma unidade hospitalar privada de Maringá-PR, para verificar a aceitação desta pelos pacientes. Foram avaliadas pacientes internadas do sexo feminino, para as intervenções gastronômicas utilizaram técnicas quanto a cortes; métodos de cocção e preparo; combinação de cores com legumes e hortaliças variadas; utilização de ervas aromáticas (salsinha desidratada, manjericão, semente de gergelim, cebola em flocos, mostarda em grão), e apresentação dos pratos. As participantes relataram que a forma de apresentação do prato influenciou o aumento do apetite e a satisfação com a refeição. Com relação ao sabor, à refeição proposta apresentou maior adequação (100\%) do uso de temperos quando comparada a refeição sem as intervenções (65,3\%). Além disso, a refeição proposta apresentou maior adequação em relação a temperatura dos alimentos servidos.

A Tabela 1 sintetiza os resultados dos estudos incluídos nesta revisão, onde pode-se observar os principais fatores que levam a baixa aceitação da dieta, bem como as aplicações gastronômicas utilizadas para melhorar a aceitação e ingestão de alimentos pelo paciente hospitalizado. 
Tabela 1: Fatores que levam a baixa aceitação da dieta e aplicações gastronômicas utilizadas para aumentar ingestão alimentar: síntese dos estudos incluídos na revisão.

\begin{tabular}{|c|c|c|c|c|c|c|c|}
\hline População & Objetivo & Desenho do estudo & $\begin{array}{l}\text { Protocolo de avaliação } \\
\text { do risco nutricional e } \\
\text { do estado nutricional }\end{array}$ & $\begin{array}{l}\text { Protocolo de } \\
\text { avaliação da } \\
\text { aceitação das } \\
\text { dietas } \\
\text { hospitalares }\end{array}$ & Ação/Intervenção & Principais resultados & Referência \\
\hline $\begin{array}{l}\text { Pacientes com } \\
\text { câncer, acima de } \\
18 \text { anos de ambos } \\
\text { os gêneros, } \\
\text { admitidos na } \\
\text { Unidade de } \\
\text { Oncologia/ } \\
\text { Hematologia de } \\
\text { um hospital } \\
\text { terciário em Porto } \\
\text { Alegre (RS) } \\
(\mathrm{n}=100) .\end{array}$ & $\begin{array}{l}\text { Verificar a aceitação } \\
\text { de dietas } \\
\text { hospitalares, em } \\
\text { relação ao estado } \\
\text { nutricional. }\end{array}$ & Estudo transversal & $\begin{array}{l}\text { Risco Nutricional: } \\
\text { Adultos (Nutritional } \\
\text { Risk Screening) e } \\
\text { Idosos (Miniavaliação } \\
\text { Nutricional) } \\
\text { Estado nutricional: } \\
\text { métodos objetivos } \\
\text { (IMC) e métodos } \\
\text { subjetivos (ASG-PPP) }\end{array}$ & $\begin{array}{l}\text { Índice de resto- } \\
\text { ingestão }\end{array}$ & - & $\begin{array}{l}\text { Elevado índice resto- } \\
\text { ingestão, sendo maior } \\
\text { percentual entre pacientes } \\
\text { desnutridos. } \\
\text { O arroz e a carne tiveram } \\
\text { maior rejeição. }\end{array}$ & $\begin{array}{l}\text { Ferreira, } \\
\text { Guimarães \& } \\
\text { Marcadenti, } 2013\end{array}$ \\
\hline $\begin{array}{l}\text { Pacientes com } \\
\text { idade acima de } 18 \\
\text { anos de ambos os } \\
\text { gêneros internados } \\
\text { no Hospital } \\
\text { Universitário } \\
\text { Sohag }(n=1015) \text {. }\end{array}$ & $\begin{array}{l}\text { Determinar a } \\
\text { satisfação com os } \\
\text { serviços de } \\
\text { alimentação } \\
\text { fornecidos pelo } \\
\text { Hospital. }\end{array}$ & Estudo transversal & - & $\begin{array}{l}\text { Questionário de } \\
\text { Satisfação do } \\
\text { Paciente do } \\
\text { ACHFPSQ } \\
\text { (Acute Care } \\
\text { Hospital } \\
\text { Foodservice). }\end{array}$ & - & $\begin{array}{l}\text { A insatisfação pelo serviço } \\
\text { foi percebida por mais de } \\
\text { um terço dos pacientes. } \\
\text { Cerca de } 73 \% \text { dos } \\
\text { entrevistados relataram não } \\
\text { consumir toda a quantidade } \\
\text { servida. }\end{array}$ & $\begin{array}{l}\text { Al-Torky et al., } \\
2016\end{array}$ \\
\hline $\begin{array}{l}\text { Pacientes adultos e } \\
\text { idosos de ambos os } \\
\text { sexos com } \\
\text { prescrição de dieta }\end{array}$ & $\begin{array}{l}\text { Verificar a aceitação } \\
\text { da dieta hipossódica } \\
\text { e o estado } \\
\text { nutricional. }\end{array}$ & Estudo transversal & $\begin{array}{l}\text { Estado nutricional: } \\
\text { métodos objetivos } \\
\text { (IMC) }\end{array}$ & $\begin{array}{l}\text { Questionário } \\
\text { sobre dados } \\
\text { sociodemográ- } \\
\text { ficos, hábitos }\end{array}$ & - & $\begin{array}{l}\text { A dieta hipossódica foi } \\
\text { considerada com sabor } \\
\text { agradável para } 81,8 \% \text { dos } \\
\text { internados, mas apresentou }\end{array}$ & $\begin{array}{l}\text { Casado \& } \\
\text { Barbosa, } 2015\end{array}$ \\
\hline
\end{tabular}




\begin{tabular}{|c|c|c|c|c|c|c|c|}
\hline $\begin{array}{l}\text { hipossódica, } \\
\text { internados em } \\
\text { Hospital Público } \\
\text { de Goiânia, Goiás } \\
(n=33) \text {. }\end{array}$ & & & & \multicolumn{2}{|l|}{$\begin{array}{l}\text { alimentares e } \\
\text { avaliação da dieta } \\
\text { hospitalar. } \\
\text { Técnica de } \\
\text { observação direta } \\
\text { para a estimativa } \\
\text { visual da porção } \\
\text { consumida. }\end{array}$} & $\begin{array}{l}\text { baixa ingestão. } \\
\text { Mais da metade dos } \\
\text { pacientes consumiam nada } \\
\text { ou menos da metade da } \\
\text { quantidade oferecida } \\
\text { (almoço } 51,51 \% \text { e jantar } \\
60,6 \%) \text {. }\end{array}$ & \\
\hline $\begin{array}{l}\text { Pacientes com } \\
\text { idade entre } 21 \text { e } 92 \\
\text { anos, de ambos os } \\
\text { gêneros, internados } \\
\text { em um hospital } \\
\text { público de } \\
\text { Goiânia-GO } \\
(\mathrm{n}=212) .\end{array}$ & $\begin{array}{l}\text { Avaliar a satisfação } \\
\text { dos pacientes em } \\
\text { relação aos tipos de } \\
\text { dietas. }\end{array}$ & $\begin{array}{l}\text { Estudo descritivo, } \\
\text { observacional e } \\
\text { qualitativo }\end{array}$ & - & $\begin{array}{l}\text { Escala hedônica } \\
\text { de cinco pontos } \\
\text { (temperatura, } \\
\text { sabor, odor, } \\
\text { quantidade e } \\
\text { aparência da } \\
\text { refeição). }\end{array}$ & - & $\begin{array}{l}\text { As dietas líquida, pastosa e } \\
\text { hipossódica apresentaram } \\
\text { maior insatisfação quando } \\
\text { comparadas a dieta livre. } \\
\text { A dieta hipossódica foi } \\
\text { considerada insatisfatória } \\
\text { no quesito sabor/tempero. }\end{array}$ & $\begin{array}{l}\text { Mendes, } \\
\text { Machado \& } \\
\text { Abreu, } 2019\end{array}$ \\
\hline $\begin{array}{l}\text { Pacientes } \\
\text { internados adultos } \\
\text { e idosos de ambos } \\
\text { os sexos, com } \\
\text { prescrição de dieta } \\
\text { oral para diabetes } \\
\text { mellitus ( } \mathrm{n}=115) \text {. }\end{array}$ & $\begin{array}{l}\text { Avaliar a } \\
\text { aceitabilidade e o } \\
\text { desperdício da } \\
\text { refeição do almoço e } \\
\text { caracterizar os } \\
\text { aspectos interferem } \\
\text { no consumo. }\end{array}$ & $\begin{array}{l}\text { Estudo descritivo } \\
\text { quantitativo }\end{array}$ & - & $\begin{array}{l}\text { Índice de resto- } \\
\text { ingestão }\end{array}$ & - & $\begin{array}{l}\text { Baixa variedade das } \\
\text { preparações, tamanho da } \\
\text { porção inadequada. } \\
\text { Os alimentos menos } \\
\text { consumidos foram arroz, } \\
\text { brócolis, feijão, carnes, } \\
\text { berinjela, cenoura e chuchu } \\
\text { cozidos. }\end{array}$ & Rigo et al., 2020 \\
\hline $\begin{array}{l}\text { Pais e responsáveis } \\
\text { por crianças } \\
\text { internadas no setor } \\
\text { oncológico }(n=17) \text {. }\end{array}$ & $\begin{array}{l}\text { Avaliar a percepção } \\
\text { de pais e } \\
\text { responsáveis quanto } \\
\text { à alimentação } \\
\text { hospitalar. }\end{array}$ & Estudo transversal & - & $\begin{array}{l}\text { Questionário com } \\
\text { perguntas sobre as } \\
\text { refeições, } \\
\text { sintomas que } \\
\text { impactaram no } \\
\text { consumo e ações } \\
\text { da equipe do } \\
\text { serviço. }\end{array}$ & - & $\begin{array}{l}\text { A temperatura, quantidade, } \\
\text { adaptação às preferências } \\
\text { dos pacientes, adaptação } \\
\text { aos sintomas e aparência } \\
\text { do prato influenciam na } \\
\text { aceitação alimentar. }\end{array}$ & $\begin{array}{l}\text { Fernandes \& } \\
\text { Spinelli, } 2020\end{array}$ \\
\hline $\begin{array}{l}\text { Pacientes } \\
\text { internados em um } \\
\text { hospital localizado } \\
\text { no Rio de Janeiro }\end{array}$ & $\begin{array}{l}\text { Avaliar a qualidade } \\
\text { das dietas } \\
\text { hospitalares a partir } \\
\text { da validação de }\end{array}$ & Estudo descritivo & - & $\begin{array}{l}\text { ESQ - Avaliação } \\
\text { da qualidade } \\
\text { sensorial das } \\
\text { refeições }\end{array}$ & - & $\begin{array}{l}\text { Elevado índice resto- } \\
\text { ingestão. Falta de } \\
\text { padronização do } \\
\text { porcionamento das }\end{array}$ & Silva et al., 2021 \\
\hline
\end{tabular}




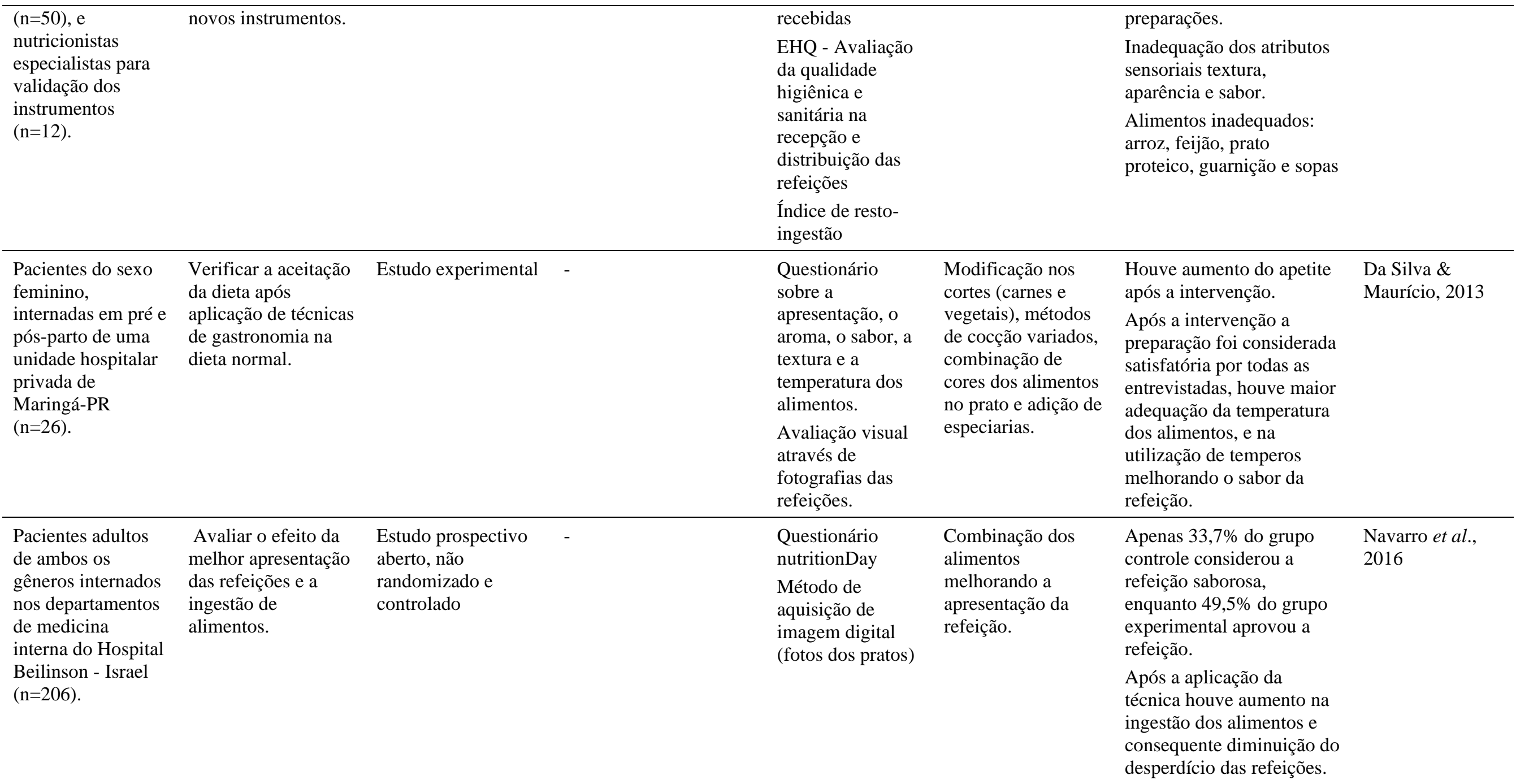




\begin{tabular}{|c|c|c|c|c|c|c|c|}
\hline $\begin{array}{l}\text { Pacientes adultos } \\
\text { com câncer, com } \\
\text { prescrição de dieta } \\
\text { pastosa em um } \\
\text { hospital } \\
\text { filantrópico de } \\
\text { Belo Horizonte, } \\
\text { MG }(n=35) \text {. }\end{array}$ & $\begin{array}{l}\text { Avaliar a eficácia da } \\
\text { aplicação de técnicas } \\
\text { gastronômicas para } \\
\text { aumentar a } \\
\text { satisfação. }\end{array}$ & Estudo experimental & & $\begin{array}{l}\text { Escala hedônica } \\
\text { de } 9 \text { pontos } \\
\text { Mensuração do } \\
\text { peso por meio de } \\
\text { balança eletrônica }\end{array}$ & $\begin{array}{l}\text { Técnicas de } \\
\text { decoração nos } \\
\text { purês, combinação } \\
\text { de cores. }\end{array}$ & $\begin{array}{l}\text { A classificação de bom e } \\
\text { excelente aumentou após a } \\
\text { intervenção. } \\
\text { Aumento das notas nos } \\
\text { atributos de aparência e } \\
\text { sabor. }\end{array}$ & $\begin{array}{l}\text { Lages, Soares \& } \\
\text { Ribeiro, } 2013\end{array}$ \\
\hline $\begin{array}{l}\text { Pacientes adultos } \\
\text { de ambos os } \\
\text { gêneros, internados } \\
\text { em um Hospital } \\
\text { Filantrópico - MG, } \\
\text { Brasil (n=97). }\end{array}$ & $\begin{array}{l}\text { Avaliar aceitação da } \\
\text { dieta após } \\
\text { implementação de } \\
\text { técnicas } \\
\text { gastronômicas. }\end{array}$ & Estudo experimental & & $\begin{array}{l}\text { Índice Resto- } \\
\text { Ingestão } \\
\text { Escala hedônica } \\
\text { de } 9 \text { pontos } \\
\text { Escala hedônica } \\
\text { facial de } 7 \text { pontos }\end{array}$ & $\begin{array}{l}\text { Adição de } \\
\text { condimentos e } \\
\text { ervas decorativas, } \\
\text { arroz formatado } \\
\text { com aro, vegetais } \\
\text { em formatos } \\
\text { decorativos, } \\
\text { variação nos } \\
\text { métodos de cocção. }\end{array}$ & $\begin{array}{l}\text { Aumento na adequação do } \\
\text { índice resto ingestão após } \\
\text { as técnicas. Houve } \\
\text { aumento das notas } \\
\text { atribuídas após a } \\
\text { intervenção nos quesitos } \\
\text { cor, sabor, aroma, textura e } \\
\text { temperatura. }\end{array}$ & $\begin{array}{l}\text { Souza, Gontijo \& } \\
\text { Almeida, } 2017\end{array}$ \\
\hline $\begin{array}{l}\text { Pacientes } \\
\text { hospitalizados } \\
\text { adultos e idosos de } \\
\text { ambos os sexos } \\
\text { com prescrição de } \\
\text { dieta hipossódica. } \\
(\mathrm{n}=90)\end{array}$ & $\begin{array}{l}\text { Avaliar aumento da } \\
\text { ingestão alimentar } \\
\text { após aplicação de } \\
\text { técnicas } \\
\text { gastronômicas. }\end{array}$ & $\begin{array}{l}\text { Estudo } \\
\text { experimental, do } \\
\text { tipo ensaio clínico } \\
\text { não randomizado, } \\
\text { controlado e cego }\end{array}$ & & $\begin{array}{l}\text { Foi estabelecido a } \\
\text { gramatura média } \\
\text { de oferta, rejeição } \\
\text { e ingestão de } \\
\text { alimentos através } \\
\text { da mensuração do } \\
\text { peso com uma } \\
\text { balança } \\
\text { eletrônica. }\end{array}$ & $\begin{array}{l}\text { Carnes, } \\
\text { leguminosas e } \\
\text { acompanhamentos } \\
\text { com adição de ervas } \\
\text { aromáticas e } \\
\text { especiarias } \\
\text { (manjericão, } \\
\text { alecrim e orégano } \\
\text { secos, alho fresco, } \\
\text { louro em pó, ervas } \\
\text { finas secas: salsa, } \\
\text { cebolinha, estragão, } \\
\text { cerefólio). }\end{array}$ & $\begin{array}{l}\text { A aceitação da dieta } \\
\text { modificada foi semelhante } \\
\text { à aceitação da dieta antes } \\
\text { da intervenção. }\end{array}$ & $\begin{array}{l}\text { Sousa, Alencar \& } \\
\text { Trindade, } 2014\end{array}$ \\
\hline
\end{tabular}


Research, Society and Development, v. 10, n. 5, e42510515138, 2021

(CC BY 4.0) | ISSN 2525-3409 | DOI: http://dx.doi.org/10.33448/rsd-v10i5.15138

\section{Conclusão e Perspectivas}

A desnutrição hospitalar é uma condição multifatorial, que tem como consequência a elevação da taxa de morbidade, mortalidade, do tempo de internação, bem como agravo do estado clínico do paciente. Diante do crescente número de pacientes com desnutrição hospitalar em todo mundo, é de suma importância que haja o acompanhamento constante dos pacientes, a aplicação de novas técnicas e abordagens voltadas para a dietoterapia, para a construção de um olhar humanizado e de novas perspectivas acerca da terapia nutricional.

A baixa aceitação de dietas hospitalares favorece a redução da ingestão de alimentos, contribuindo para o agravamento do estado nutricional de pacientes internados, e está relacionada a diversos fatores, como anorexia, alterações gastrointestinais, capacidade reduzida de deglutição ou ingestão dos alimentos ocasionados por problemas de mastigação, disfagia, alteração no processo de digestão e pelas características sensoriais dos alimentos servidos.

Com relação às características sensoriais, os principais motivos que interferem na aceitação da dieta são a inadequação de temperatura das refeições servidas, apresentação da preparação, baixa variedade de alimentos, porcionamento acima do adequado, falta de sabor/tempero, falta de padronização das preparações. Nesse sentido, a aplicação de estratégias de gastronomia hospitalar que possibilitem a melhoria das características sensoriais das preparações são ferramentas importantes para aumentar a aceitação das preparações oferecidas aos pacientes, favorecendo a manutenção e/ou recuperação do estado nutricional do paciente, bem como a redução do desperdício de alimentos.

Por fim, recomenda-se que sejam realizados mais estudos sobre essa temática visto que a mesma ainda é pouco explorada pela literatura científica, para que sejam avaliadas e desenvolvidas novas estratégias voltadas para a gastronomia hospitalar aplicadas à dietoterapia.

\section{Referências}

Al-Torky, M., Mohamed, E. A., Yousef, F. M. \& Ali, N. A. (2016). Inpatients' satisfaction with food services in Sohag University Hospital. The Egyptian Journal Of Community Medicine, 34(2), 33-45. http://dx.doi.org/10.21608/ejcm.2016.651

Augustini, V. C. M.; Kishimoto, P; Tescaro, T.C. \& Almeida, F. Q. A. (2008). Avaliação do índice de resto-ingesta e sobras em unidade de alimentação e nutrição (UAN) de uma empresa metalúrgica na cidade de Piracicaba / SP. Revista Simbio-Logias, Botucatu, 1(1), 99- 110. https://www.ibb.unesp.br/Home/ensino/departamentos/educacao/revistasimbio-logias/v1-nr1-2008/avaliacao_indice_resto-ingesta.pdf

Barker, L., Gout, B. \& Crowe, T. (2011). Hospital Malnutrition: Prevalence, Identification and Impact on Patients and the Healthcare System. International Journal of Environmental Research and Public Health, 8(2), 514-527. http://dx.doi.org/10.3390/ijerph8020514

Beser, O. F., Cokugras F.C., Erkan T., Kutlu T. \& Yagci, R.V. (2018). Evaluation of malnutrition development risk in hospitalized children. Nutrition. 48,4047 https://doi.org/10.1016/j.nut.2017.10.020

Cardenas, D., Bermúdez, C., Pérez, A., Diaz, G., Cortés, L. Y., Contreras, C. P., Pinzón-Espitia, O. L., Gómez, G., González, M. C., Fantin, R., Gutierrez, J., Sulz, I., Tarantino, S. \& Hiesmayr, M. (2021). Are traditional screening tools adequate for monitoring the nutrition risk of in-hospital patients? An analysis of the nutritionDay database. JPEN J Parenter Enteral Nutrition. https://doi.org/10.1002/jpen.2085

Caruso, L., Simony, R. F. \& Silva, A. D. (2005). Dietas Hospitalares: uma Abordagem na Prática Clínica. Atheneu: 148 páginas

Casado, A. V. D. M. \& Barbosa, L. S. (2015). Aceitação de dieta hipossódica e estado nutricional de pacientes internados em hospital público de Goiânia. $O$ Mundo da Saúde, São Paulo, 39(2), 188-194. http://dx.doi.org/10.15343/0104-7809.20153902188194.

Cheng, J., Witney-Cochrane, K., Cunich, M., Ferrie, S. \& Carey, S. (2019). Defining and quantifying preventable and non-preventable hospital-acquired malnutrition-A cohort study. Nutrition \& Dietetics. 76(5), 620-627. http://dx.doi.org/10.1111/1747-0080.12553

Coloço, R. B., Holanda, L. B. \& Portero-MCLellan, K. C. (2009). Determinantes do grau de satisfação de pacientes internados referente a refeições oferecidas em um hospital universitário. Revista Ciências Médicas, 3(18), 121-130

Correia, M. I. T. D., Perman, M. I., Waitzberg, D. L. (2017). Hospital malnutrition in Latin America: A systematic review. Clinical Nutrition, $36(4), 958-967$. http://dx.doi.org/10.1016/ j.clnu.2016.06.025 
Research, Society and Development, v. 10, n. 5, e42510515138, 2021

(CC BY 4.0) | ISSN 2525-3409 | DOI: http://dx.doi.org/10.33448/rsd-v10i5.15138

Da Silva, S. M. \& Maurício, A. A. (2013). Gastronomia hospitalar: um novo recurso para melhorar a aceitação de dietas. Conscientiae Saúde, 12(1), 17-27. http://dx.doi.org/10.5585/conssaude.v12n1.3497

Fernandes, R. C. S. \& Spinelli, M. G. N. (2020). Percepção de pais e responsáveis por crianças diagnosticadas com câncer: a gastronomia hospitalar em foco. Nutricion Clinica y Dietetica Hospitalaria, 40(1), 20-24. http://dx.doi.org/10.12873/401fernandes.

Fernández-Martínez, B., Alguacil-Pau, A. I., Crespo-Sevillac, R. \& García-Veja, A. (2013). Predictores de la satisfacción de los pacientes con la alimentación de un hospital público de Madrid. Revista de Calidad Asistencial, 28(3):155-162. http://dx.doi.org/10.1016/j.cali.2012.09.005

Ferreira, D., Guimarães, T. G. \& Marcadenti, A. (2013). Aceitação de dietas hospitalares e estado nutricional entre pacientes com câncer. Einstein (São Paulo), 11(1), 41-46. Einstein (São Paulo). http://dx.doi.org/10.1590/s1679-45082013000100008

García, M. G. \& La Hera, A. R. L. H. (2018). Dietética hospitalaria y gastronomía saludable. Nutrición Hospitalaria, 35(4), 140-145. http://dx.doi.org/10.20960/nh.2140

Kang, M. C., Kim, J. H., Ryu, S. W., Moon, J. Y., Park, J. H., Park, J. K., Park, J. H., Baik, H. W., Seo, J. M., Son, M. W., Song, G. A., Shin, D. W., Shin, Y. M., Ahn, H. Y., Yang, H. K., Yu, H. C., Yun, I. J., Lee, J. G., Lee, J. M., Lee, J. H., Lee, T. H., Yim, H., Jeon, H. J., Jung, K., Jung, M. R., Jeong, C. Y., Lim, H. S. \& Hong, S. K. (2018). Prevalence of malnutrition in hospitalized patients: A multicenter cross-sectional study. Journal of Korean Medical Science, 33(2), 1-10. http://dx.doi.org/10.3346/ jkms.2018.33.e10

Konturek, P. C., Herrmann, H. J., Schink, K., Neurath, M. F. \& Zopf, Y. (2015). Malnutrition in Hospitals: It Was, Is Now, and Must Not Remain a Problem! Med Sci Monit. (21), 2969-75. http://dx.doi.org/10.12659 / MSM.894238

Lages, P. C., Soares, L. S. \& Ribeiro, R. C. (2013) A gastronomia como proposta de qualificação dietética das refeições hospitalares pastosas: análise, intervenção e avaliação. Alimentos e Nutrição Araraquara, 24(1), 93-100. http://serv-bib.fcfar.unesp.br/seer/index.php/alimentos/article/viewFile/2416/2416

Leandro-merhi, V. A., Srebernich, S. M., Gonçalves, G. M. S., \& Aquino, J. L. B. (2015). In-hospital weight loss, prescribed diet and food acceptance. Abcd. Arquivos Brasileiros de Cirurgia Digestiva. 28(1), 8-12, 2015. http://dx.doi.org/10.1590/s0102-67202015000100003

Lew, C. C. H., Yandell, R., Fraser, R. J. L., Chua, A. P., Chong, M. F. F. \& Miller, M. (2017). Association Between Malnutrition and Clinical Outcomes in the Intensive Care Unit: A Systematic Review. JPEN J Parenter Enteral Nutrition 41(5), 744-758. https://doi:10.1177/0148607115625638

Lima, A. M., Gamallo, S. M. M., \& Oliveira, F. L. C. (2010). Desnutrição energético-proteica grave durante a hospitalização: aspectos fisiopatológicos e terapêuticos. Revista. Paulista de Pediatria 28(3), 353-361. https://doi.org/10.1590/S0103-05822010000300015

Lorenzo, A. G., Hernández, J. A., Planas, M., Burgos, R., \& Araujo, K. (2011). Multidisciplinary consensus on the approach to hospital malnutrition in Spain. Nutrición Hospitalaria, 26(4), 701-710. http://scielo.isciii.es/scielo.php?script=sciarttext\&pid= S0212-16112011000400006\&lng=es\&tlng=

Lovesley, D., Parasuraman, R. \& Ramamurthy, A. (2019). Combating hospital malnutrition: Dietitian-led quality improvement initiative. Revista Clinical Nutrition Espen, 30, 19-25. https://doi.org/10.1016/j.clnesp.2019.02.011

Maeda, K., Ishida,Y., Nonogaki, T., Shimizu, A., Yamanaka, Y., Matsuyama, R., Kato, R., Mori, N. (2019). Carga do consumo pré-mórbido de dietas modificadas com textura na vida diária sobre o estado nutricional e os resultados da hospitalização. J Nutr Health Aging 23, 973-978. https://doi.org/10.1007/s12603-019-12373

Mahan, L. K. \& Raymond, J. L. (2018). Krause Alimentos, Nutrição e Dietoterapia. (14a ed), Elsevier, 1123 páginas

Marliere, A., Cremonezi, C., Faccioli, J. M., Garcia, P. \& Wanda, R. (2016). Atualidades em alimentação e nutrição hospitalar. Atheneu: 544 páginas

Martins, P. \& Baratto, I. (2018). Gastronomia hospitalar: treinamento em bases de cozinha. Revista Brasileira de Obesidade, Nutrição e Emagrecimento, 12(69), 110-117.

McCarthy, A., Delvin, E., Marcil, V., Belanger, V., Marchand, V., Boctor, D., Rashid, M., Noble, A., Davidson, B., Groleau, V., Spahis, S., Roy, C. \& Levy, E. (2019). Prevalence of Malnutrition in Pediatric Hospitals in Developed and In-Transition Countries: The Impact of Hospital Practices. Nutrients, 11(2), 118. https://doi.org/10.3390/nu11020236

Mendes, M. S. A., Machado, C. C. B. \& Abreu, V. S. (2019). Pesquisa de satisfação com dietas hospitalares servidas no almoço da clínica médica de um hospital público de Goiânia, GO. Demetra: Alimentação, Nutrição \& Saúde, 14, 1-11. http://dx.doi.org/10.12957/demetra.2019.38989.

Moriana, A. M., Civera, M., Artero, A., Real, J. T., Caro, J., Ascaso, J. F. \& Martinez-Valls, J. F. (2014). Validez de la valoración subjetiva global como método de despistaje de desnutrición hospitalaria. Prevalencia de desnutrición en un hospital terciario. Endocrinología y Nutrición 61(4), 184-189. http://dx.doi.org/10.1016/j.endonu.2013.10.006

Nascimento, T., Moreira, D. F., Carvalho, R. C. R., Pereira, M. A. O., Pereira, E. A. A. \& Vilela, B. S. (2017). Aceitabilidade das dietas orais de um hospital do Sul de Minas. Revista UIIPS, 5(5), 141-148. https://doi.org/10.25746/ruiips.v5.i5.14551

Navarro, D. A. Boaz, M., Krause, I., Elis, A., Chernov, K., Giabra, M., Levy, M., Giboreau, A., Kosak, S., Mouhieddine, M. \& Singer, P. (2016). Improved meal presentation increases food intake and decreases readmission rate in hospitalized patients. Clinical nutrition, 35(5), 1153-1158. http://dx.doi.org/10.1016/j.clnu.2015.09.012 0261-5614

Novinski, A. F., Araújo, G. C., \& Baratto, I. (2017). Resto ingesta em uma unidade de alimentação e nutrição hospitalar na cidade de Pato Branco-PR. Revista Brasileira de Obesidade, Nutrição e Emagrecimento, 11(66), 451-458. http://www.rbone.com.br/index.php/rbone/article/view/569 
Oliveira, E. J. C., Oliveira, T. C. \& Santos, V. S. (2020). “Dentro das condições que a gente tem”: Percepções de nutricionistas sobre gastronomia em hospital universitário. Revista de Enfermagem e Atenção À Saúde, 9(2), 28-38. http://dx.doi.org/10.18554/reas.v9i2.3966.

Orlandoni, P., Peladic, N. J., Cola, C., Venturini, C., Costantini, A., Giorgini, N., Basile, R., Sparvoli, D., \& David, S. (2018). Hospital acquired malnutrition in orally fed geriatric patients: What's the role of a hospital dietetics and food service?. Progress in Nutrition. 20(2), 225-231. https://doi.org/10.23751/pn.v20i2-S.5780

Pinto, C. C. \& Alves, E. A. (2020). A gastronomia no contexto da hotelaria hospitalar: um estudo de caso na cidade do Rio de Janeiro, RJ, Brasil. RahisRevista de Administração Hospitalar e Inovação em Saúde, 14(2),1-13. http://dx.doi.org/10.21450/rahis.v14i2.3844.

Rattray, M.; Desbrow, B. \& Roberts, S. (2017). Comparing nutritional requirements, provision and intakes among patients prescribed therapeutic diets in hospital: an observational study. Nutrition, (39), 50-56. http://dx.doi.org/10.1016/j.nut.2017.03.006 0899-9007

Rigo, A. M., Strashurg, V. J., Goularte, J. F., Escobar, M. \& Jochims, M. K. (2020). Aceitabilidade e fatores associados ao consumo dietético em pacientes diabéticos de um hospital público universitário. Demetra: Alimentação, Nutrição \& Saúde, 15, 1-13. http://dx.doi.org/10.12957/demetra.2020.51595

Rolim, P. M., Souza, K. M., Filgueira, L. P., \& Silva, L. C. (2011). Apresentação da refeição versus desperdício de alimentos na alimentação de pacientes oncológicos. Alim. Nutr. 22(1), 137-142.

Ross, A. C., Caballero, B. M. D., Cousins, R. J., Tucker, K. L. \& Ziegler, T. R. (2016). Nutrição Moderna de Shils na Saúde e na Doença. São Paulo: Manole, 1660 páginas

Silva, E. P. \& Tiengo, A. (2014). Perfil Nutricional de Crianças Hospitalizadas e sua Relação com o Período de Internação em um Hospital de Ensino no Sul de Minas Gerais. Revista Ciências em Saúde. 4 (4), 61-72. https://doi.org/10.21876/rcsfmit.v4i4.265

Saengnipanthkul, S., Chongviriyaphan, N., Densupsoontorn, N., Apiraksakorn, A., Chaiyarit, J., Kunnangja, S., Wongpratoom, S., Papakhee, S., Detamnatkul, W., Monwiratkul, J., Saengpanit, P., Limthongthang, P., \& Panthongviriyakul, C. (2021). Hospital-acquired malnutrition in paediatric patients: a multicentre trial focusing on prevalence, risk factors, and impact on clinical outcomes. European Journal of Pediatrics. https://doi.org/10.1007/s00431-02103957-9

Schindler, K., Kosak S., Schütz, T., Volkert, D., Hürlimann, B., Ballmer, P. \& Hiesmayr M. (2014). NutritionDay- an annual cross-sectional audit of nutrition in healthcare. Ther Umsch. 71(3), 127-133. http://dx.doi.org/10.1024/0040-5930/a000493

Silva, M. P., Brito, P. D., Silva, F. M., Almeida, C. F., Silva, P. S. \& Colares, L. G. T. (2021). Aprimoramento e validação de conteúdo de instrumentos para avaliar a qualidade de dietas hospitalares. Demetra: Alimentação, Nutrição \& Saúde, 16, 1-15. http://dx.doi.org/10.12957/demetra.2021.52365

Sousa, A. A., Alencar, M. L. A. \& Trindade, E. B. S. M. (2014). Especiarias e condimentos aumentam a ingestão alimentar de pacientes com dieta hipossódica? Demetra: Alimentação, Nutrição \& Saúde, 9(3), 795-809. http://dx.doi.org/10.12957/demetra.2014.11224.

Souza, M. D. \& Nakasato, M. (2011) A gastronomia hospitalar auxiliando na redução dos índices de desnutrição entre pacientes hospitalizados. $O$ mundo da saúde, 35(2), 208-214

Souza, S. V., Gontijo, M. C. F. \& Almeida, M. E. F. (2017). Efeito da gastronomia na aceitabilidade de dietas hospitalares. Nutrición clínica y Dietética Hospitalaria, 37(3), 17-22. http://dx.doi.org/10.12873/373souza

Syuhada, K., Wanda, D., Nur'aini, R., Ardiantari, C. \& Susilo, A. (2020). Statistical Risk Characteristics and Risk Scoring of Hospital-Acquired Malnutrition for Pediatric Patients. Journal of Nutrition and Metabolism. https://doi.org/10.1155/2020/4305487

Taldivo, B. P. \& Santos, M. C. T. (2016). Gastronomia hospitalar. Revista Conexão Eletrônica, 13(1), 1-8.

Theilla, M., Grinev, M., Kosak, S., Hiesmayr, M. \& Singer, P. (2015). Fight against malnutrition: The results of a $2006 \mathrm{e} 2012$ prospective national and global nutritionDay survey. Clinical Nutrition ESPEN, (10), 77-82. https://doi.org/10.1016/j.clnesp.2015.01.002

Toledo, D. O., Piovacari, S. M. F., Horie, L. M., Matos, L. B. N., Castro, M. G., Ceniccola, G. D., Corrêa, F. G., Giacomassi, I. W. S., Barrére, A. N., Campos, L. F., Verotti, C. C. G., Matsuba, C. S. T., Gonçalves, R. C., Falcão, H., Dib, R., Lima, T. E. C., Souza, I. A. O., Gonzalez, M. C. \& Correia, M. I. D. (2018). Campanha "Diga não à desnutrição": 11 passos importantes para combater a desnutrição hospitalar. BRASPEN J 33(1), 86-100

Waitzberg, D. L. Caiaffa, W. T. \& Correia, M. I. T. D. (2001). Hospital malnutrition: the Brazilian national survey (IBRANUTRI): a study of 4000 patients. Nutrition, 17(7-8), 573-580.

World Health Organization (WHO) (1997). Programme of Nutrition WHO Global Database on Child Growth and Malnutrition. Geneva. http://apps.who.int/iris/bitstream/10665/63750/1/WHO/_NUT_97.4.pdf

Zheng, H., Huang, Y., Shi, Y., Chen, W., Yu, J. \& Wang, X. (2016). Nutrition status, nutrition support therapy, and food intake are related to prolonged hospital stays in China: results from the Nutrition Day 2015 Survey. ANNALS OF NUTRITION AND METABOLISM. 69(3-4), 215-225. https://doi.org/10.1159/000451063 